\title{
Adherence to guidelines and protocols in the prehospital and emergency care setting: a systematic review
}

Remco HA Ebben ${ }^{1,5^{*}}$, Lilian CM Vloet ${ }^{1,2}$, Michael HJ Verhofstad ${ }^{3}$, Sanne Meijer ${ }^{1}$, Joke AJ Mintjes-de Groot ${ }^{1}$ and Theo van Achterberg ${ }^{4}$

\begin{abstract}
A gap between guidelines or protocols and clinical practice often exists, which may result in patients not receiving appropriate care. Therefore, the objectives of this systematic review were (1) to give an overview of professionals' adherence to (inter)national guidelines and protocols in the emergency medical dispatch, prehospital and emergency department (ED) settings, and (2) to explore which factors influencing adherence were described in studies reporting on adherence. PubMed (including MEDLINE), CINAHL, EMBASE and the Cochrane database for systematic reviews were systematically searched. Reference lists of included studies were also searched for eligible studies. Identified articles were screened on title, abstract and year of publication $(\geq 1990)$ and were included when reporting on adherence in the eligible settings. Following the initial selection, articles were screened full text and included if they concerned adherence to a (inter)national guideline or protocol, and if the time interval between data collection and publication date was $<10$ years. Finally, articles were assessed on reporting quality. Each step was undertaken by two independent researchers. Thirty-five articles met the criteria, none of these addressed the emergency medical dispatch setting or protocols. Median adherence ranged from 7.8-95\% in the prehospital setting, and from 0-98\% in the ED setting. In the prehospital setting, recommendations on monitoring came with higher median adherence percentages than treatment recommendations. For both settings, cardiology treatment recommendations came with relatively low median adherence percentages. Eight studies identified patient and organisational factors influencing adherence. The results showed that professionals' adherence to (inter)national prehospital and emergency department guidelines shows a wide variation, while adherence in the emergency medical dispatch setting is not reported. As insight in influencing factors for adherence in the emergency care settings is minimal, future research should identify such factors to allow the development of strategies to improve adherence and thus improve quality of care.
\end{abstract}

Keywords: Emergency medical technicians [MeSH], Emergency medical services [MeSH], Emergency medicine $[\mathrm{MeSH}]$, Emergency nursing [MeSH], Guideline adherence [MeSH]

\footnotetext{
* Correspondence: Remco.Ebben@han.nl

${ }^{1}$ Research group for Acute Care, Faculty of Health and Social Studies, HAN

University of Applied Sciences, Verlengde Groenestraat 75, Nijmegen 6525 EJ,

The Netherlands

${ }^{5}$ Research group for Acute Care, Faculty of Health and Social Studies, HAN

University of Applied Sciences, PO Box 6960, 6503 GL, Nijmegen, The

Netherlands

Full list of author information is available at the end of the article
}

C Biomed Central

(c) 2013 Ebben et al.; licensee BioMed Central Ltd. This is an Open Access article distributed under the terms of the Creative Commons Attribution License (http://creativecommons.org/licenses/by/2.0), which permits unrestricted use, distribution, and reproduction in any medium, provided the original work is properly cited. 


\section{Introduction}

Clinical practice guidelines and protocols are developed to improve quality of care, to reduce variation of practice and to ensure that evidence is actually used when appropriate [1]. Often, these instruments are developed and disseminated by (inter)national professional organisations [2,3]. A guideline consists of systematically developed recommendations to assist practitioners and patient decisions about appropriate health care for specific clinical circumstances [4]. A guideline recommendation is defined as "any statement that promotes or advocates a particular course of action in clinical care" [5]. To assist implementation of guidelines, a protocol can be developed, which yields a specification of a guideline and exactly formulates how to act and which steps to follow [6]. Despite the existence of guidelines and protocols, a gap between recommended care and clinical practice often exists $[7,8]$. This is shown in a systematic review on the quality of health care delivered to adults in the United States [9]. Results showed that patients received $54.9 \%$ of recommended care, that the proportion of recommended care slightly differed for preventive, acute, and chronic care, and that differences were even larger for different medical functions (screening, diagnosis, treatment and follow-up).

It is suggested that effective implementation should ensure guideline adherence in practice and subsequently lead to improved patient outcomes [5]. Implementation is defined as "a planned process and systematic introduction of innovations or changes of proven value; the aim being that these are given a structural place in professional practice, in the functioning of organisations or in the health care structure" [6]. A systematic review on factors influencing implementation of clinical guidelines concluded that influencing factors were related to the used implementation strategies, and characteristics of the guidelines, professionals, patients and environment [10].

Similar to other settings, guidelines and protocols have become an important aspect of prehospital and emergency care clinical practice $[11,12]$. Yet, only few studies have investigated to what extent emergency care professionals actually adhere to these instruments [11]. When professionals do not adhere to guidelines and protocols, patients in the prehospital and emergency care settings may not receive appropriate care and quality of care can be threatened.

\section{Objective}

The first objective of this study was to present an overview of professionals' adherence to (inter)national guidelines and protocols in the emergency medical dispatch, prehospital and emergency department (ED) setting. The underlying rationale for choosing these settings is that they are often regarded as 'the chain of emergency care' and that all professionals, irrespective of setting, are expected to provide emergency care as described in guidelines and protocols. The second objective was to explore which factors influencing adherence were described in studies reporting on adherence. This insight can provide valuable input for the development of strategies to successfully implement guidelines and protocols in the emergency care settings.

\section{Methods}

A systematic review of the literature was performed. The review is reported conform the PRISMA statement (Preferred Reporting Items for Systematic Reviews and Meta-Analysis) [13].

\section{Type of studies}

All types of quantitative studies which described adherence to guidelines or protocols in the emergency medical dispatch, prehospital ambulance care and ED settings were included. Studies using self-report methods were excluded as they incorporate a risk of overestimation [14].

\section{Type of guidelines}

Studies describing adherence to (inter)national guidelines and protocols concerning all types of medical conditions in all types of emergency settings in all countries and regions within countries were included. Studies concerning local guidelines and protocols were excluded as it was unclear how they were developed and to what degree they were evidence-based.

\section{Type of outcome measures}

One of the outcome measures of the study had to include adherence quantified as percentage.

\section{Electronic searches}

PubMed (including MEDLINE), CINAHL, EMBASE and the Cochrane database for systematic reviews were searched in June 2010. Search strategies contained 'terms for professionals' AND 'terms for settings' AND 'terms for adherence' AND 'terms for guidelines/ protocols'. Full search strategies per database are given in Appendix 1. Searches were restricted by year of publication ( $\geq 1990)$. No other restrictions were used. In addition to the electronic search, we hand searched reference lists of included articles. We searched the Cochrane database for systematic reviews for both planned and completed reviews on adherence, but found none.

\section{Selection of studies}

All articles were screened on title and abstract by two independent reviewers (RE, LV) and included if the title or abstract described adherence in one of the emergency 
care settings. After initial selection, remaining articles were screened full text by researchers in two pairs (RE, $\mathrm{LV}, \mathrm{JM}, \mathrm{TvA}$ ) and were included if (a) the adherence concerned specified guidelines or protocols, and (b) if the time interval between data collection and publication date of the guideline or protocol did not exceed ten years as non-adherence with outdated recommendations might be justified in these cases. Conference abstracts, editorials, personal communications, or unpublished studies were excluded.

\section{Quality assessment}

To provide a quality indicator, two pairs of independent researchers assessed reporting quality of all included studies (RE, LV, JM, TvA). For this assessment we developed a checklist, which was based on the STROBE statement to assess the reporting of cohort and cross sectional studies [15] and the TREND statement to assess the reporting of interventional studies [16]. The checklist consisted of ten items to assess quality: (1) objective, (2) key elements, (3) setting, locations and dates, (4) eligibility criteria, (5) outcomes, (6) data sources and methods, (7) data analysis and statistical methods, (8) number of participants, (9) characteristics of participants, and (10) main results. For each item an article could score a 'described' (1 point), 'partly described' (0.5 point), or 'not described' (0 points). All included articles were rated on a scale from 1 (poor study report) to maximum 10 (excellent study report).

\section{Data extraction}

From each article (a) the number of guideline or protocol recommendations described, and (b) adherence percentages for each recommendation were extracted. In case of multiple measurements regarding one recommendation, multiple adherence percentages were extracted. In case of a pre-test post-test design for the evaluation of quality improvement, only the pre-test percentages were extracted as we focused on actual care rather than effects of quality improvement strategies. From each study, the guideline and protocol recommendations were categorised into medical condition (cardiology, pulmonology, neurology, infectious diseases, or other) and into type of medical function (diagnostic, treatment, monitoring, or organisational) (Table 1). Categorisation was done as 'medical condition' and 'medical function' have been indicated as influencing factors for guideline adherence previously [10,17]. The median adherence for each recommendation was extracted or calculated. Additionally, factors influencing adherence were extracted when a statistically significant relationship between the factor and adherence was demonstrated in the article. Nonsignificant factors are not shown. The corresponding author of one study was contacted through e-mail to clarify and confirm results.

All data were extracted by two independent researchers (RE, SM). To assess inter-rater reliability, the overall agreement percentages were calculated on number of guideline or protocol recommendations and adherence percentages. For articles concerning the prehospital care setting, these were $93 \%$ and $83 \%$ respectively, and for articles concerning the ED setting these were $90 \%$ and $85 \%$. Since the heterogeneity of study designs, guideline recommendations, medical conditions, and medical functions was substantial, a meta-analysis was not feasible. Instead, we extensively analysed the studies and conducted a qualitative synthesis.

\section{Results}

\section{Description of the studies}

The electronic search identified 30 articles meeting the inclusion criteria. In addition, another five articles were included by searching the reference lists (Figure 1). Of the included articles $(\mathrm{n}=35), 24$ used retrospective, 9 used prospective, and 2 used cross sectional methods. Eighteen studies were multicentric and seventeen were monocentric, with 31 covering adults and 4 covering children. The studies were conducted in North America $(n=19)$, Europe $(\mathrm{n}=13)$, Australia $(\mathrm{n}=2)$, and Asia $(\mathrm{n}=1)$. One study described adherence in the prehospital setting as well as in the ED setting [18] and results of this study are therefore

Table 1 Categories of guideline recommendations classified by medical function

\begin{tabular}{ll}
\hline Medical function & Examples \\
\hline Diagnostic & 1. Evaluate arterial blood gas for patients with acute exacerbations of COPD [19] \\
& 2. Obtain blood culture in case of a child with fever [42] \\
Treatment & 1. Administer benzyl penicillin if a patient has a non-blanching purpuric rash [25] \\
& 2. Administer epinephrine 1 $\mathrm{mg}$ intravenous, intraosseous or endotracheal if a patient has cardiac arrest [27] \\
Monitoring & 1. Monitor blood pressure and $\mathrm{SaO}_{2}$ at least once for a patient with cardiac arrest [26] \\
& 2. Monitor EtCO $\mathrm{CO}_{2}$ for a patient with cardiac arrest [26] \\
Organisational (referral, documentation) & 1. Refer to an allergist in case of a severe allergic reaction [49] \\
& 2. Document asthma severity (mild, moderate, severe) [35] \\
\hline
\end{tabular}




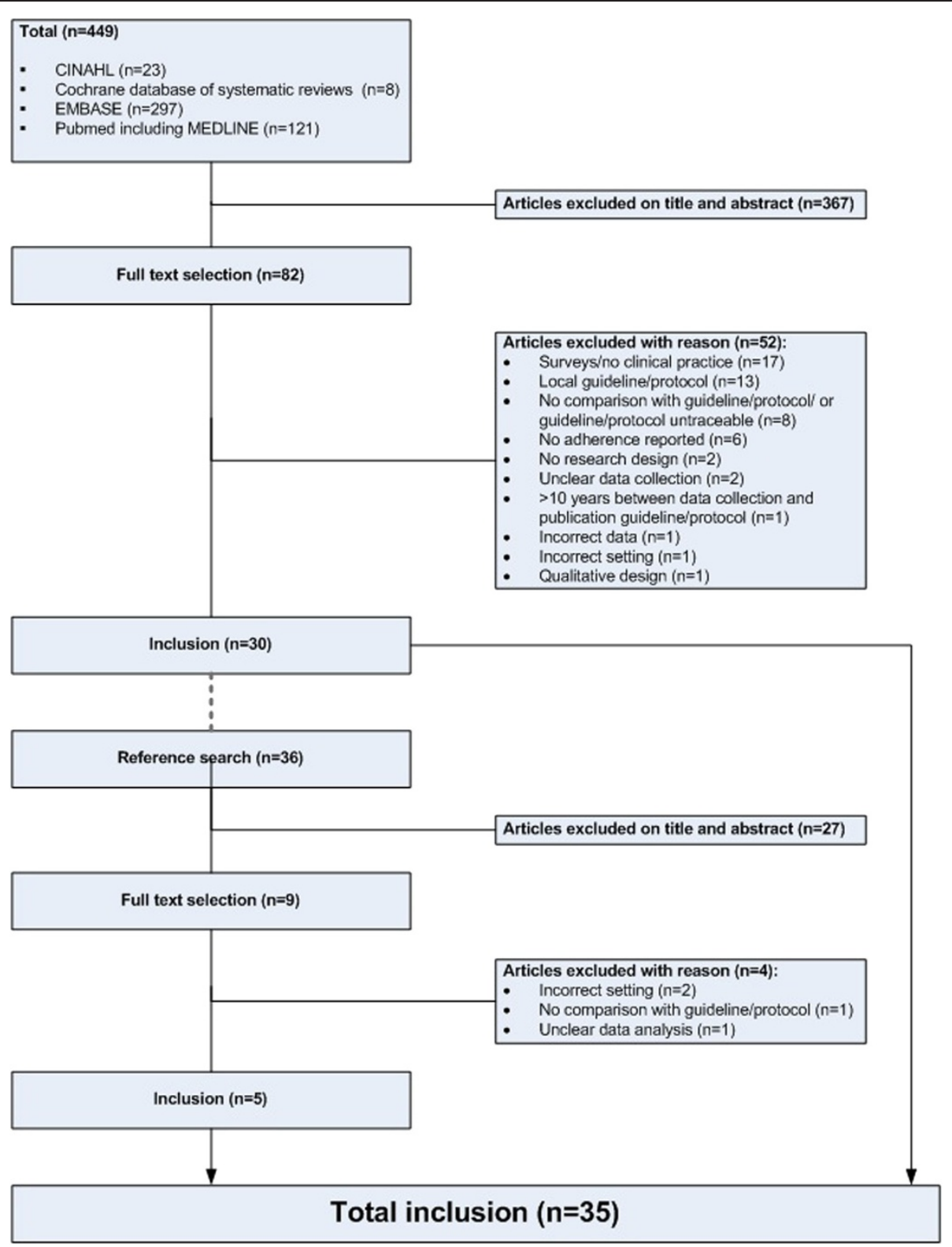

Figure 1 Inclusion of studies.

presented in both the prehospital and ED result sections. All studies described adherence to (inter)national guidelines No studies on adherence to (inter)national protocols were identified. Seven studies assessed adherence to a guideline which was not developed in their own country [18-24]. The quality assessment revealed 34 articles of excellent or good reporting quality (excellent report - ten points, very good report - nine points, good report - eight points). Only one article was of moderate reporting quality with seven points [25]. As only the reporting quality was assessed, no articles were excluded on the basis of this quality assessment. Further details of the included studies are described in Table 2.

\section{Emergency medical dispatch}

Our electronic search strategy and reference search did not identify any eligible studies in the emergency medical dispatch setting.

\section{Prehospital setting}

Ten studies were identified describing adherence to (inter) national guidelines in the prehospital setting. These guidelines covered cardiology [18,26-28], pulmonology [29], neurology [30-33], and infectious diseases [25] (Table 3). Professionals included emergency physicians, anesthesiologists, ambulance nurses, nurse anesthesists, 
Table 2 Characteristics of included studies $(n=35)$

\begin{tabular}{lllll}
\hline $\begin{array}{l}\text { First author Design } \\
\text { (Year) } \\
\text { Country }\end{array}$ & Methods & $\begin{array}{l}\text { Monocenter/ Professionals } \\
\text { multicenter }\end{array}$ & Patients & Guideline (year of publication) Quality \\
\hline
\end{tabular}

Prehospital

\section{Caulfield}

(2009)

USA

Cooke

(2005)

UK

Retrospective,
descriptive

Prehospital Monocenter: HEMS paramedics record 1 EMS

review

EMS

Retrospective, Patient Multicenter: Paramedics descriptive

report

19 EMSs

forms

$\begin{array}{llll}\text { Retrospective, } & \text { Medical } & \text { Monocenter: } & \text { Ambulance } \\ \text { descriptive } & \begin{array}{l}\text { record } \\ \text { review }\end{array} & 1 \text { EMS } & \begin{array}{l}\text { nurses EMS } \\ \text { physicians }\end{array}\end{array}$

Franschman

2009

The Netherlands

review

Retrospective,

descriptive

Prehospital Monocenter: Not specified

(2008)

UK

Jeremie
(2005)
France
Kirves
(2007)

Finland

Scliopou

(2005)

USA

Thomas

(2002)

USA

Wik

(2005) Norway/

Sweden/UK
Retrospective,

descriptive

Database

review

Multicenter:

35 EMSs

Prospective,

descriptive

Data

collection

chart

Prospective

case series
Data cards

EMS
Monocenter: HEMS nurses

1 EMS

Nurse

anesthesists

Paramedics
HEMS paramedics

100 patients with Brain Trauma Foundation

traumatic brain injury Guideline for prehospital

69 patients with Joint Royal Colleges Ambulance

suspected

meningococcal

septicemia

127 patients with Brain Trauma Foundation

traumatic brain injury Guideline for prehospital

1022 patients who received $\mathrm{O}_{2}$

143 patients who were sedated and intubated

157 patients with cardiac arrest

cardiac arrest

37 patients with

176 patients with Guidelines for Cardiopulmonary cardiac arrest

1277 patients with

ST-segment

elevation myocardial infarction UH, 8 EMSs, physicians 26 MICUs, 37 EDs, 22 CICUs

\section{Emergency Department}

Atreja

(2005)

USA
Retrospective, Chart Monocenter: Emergency descriptive review physicians
94 patients with an elevated international normalized ratio (INR) management of patients with traumatic brain injury (2007)

Liaison Committee Clinica

Guidelines for the administration

of benzyl penicillin for suspected

diagnosis of meningococcal

septicemia (2003)

management of patients with

traumatic brain injury (2007)

Dutch Ambulance Care National

Protocol (2007)

Joint Royal Colleges Ambulance

Liaison Committee Clinical

Guidelines for the administration

of oxygen (2007)

SFAR Recommendations for sedation: analgesia in out-of -emergency medicine (2000)

The Subdivision of Emergency Medicine of Finnish Society of Anaesthesiologists, Finnish Resuscitation Council and Red Cross of Finland. Resuscitation guidelines (2002)

American Heart Association Advanced cardiac Life Support Guidelines (2000)

Brain Trauma Foundation

guidelines for the Management of Severe Head Injury (1995)

Resuscitation and Emergency

Cardiovascular Care: International Consensus on Science (2000)

International guidelines for CPR and ECCL: a consensus on science (2000)

American College of Cardiology/ American Heart Association guidelines for the management of patients with acute myocardial infarction (1999)

Physicians recommendations for antithrombotic therapy for prevention and treatment of thrombosis (2001) 
Table 2 Characteristics of included studies $(\mathbf{n}=\mathbf{3 5})$ (Continued)

\begin{tabular}{|c|c|c|c|c|c|c|c|}
\hline $\begin{array}{l}\text { Clark } \\
\text { (2004) } \\
\text { USA \& Canada }\end{array}$ & $\begin{array}{l}\text { Retrospective, } \\
\text { cohort }\end{array}$ & $\begin{array}{l}\text { Medical } \\
\text { record } \\
\text { review }\end{array}$ & $\begin{array}{l}\text { Multicenter: } \\
21 \text { EDs }\end{array}$ & Not specified & $\begin{array}{l}678 \text { patients with } \\
\text { allergic reaction to } \\
\text { food }\end{array}$ & $\begin{array}{l}\text { American academy of allergy, } \\
\text { asthma, \& immunology guideline } \\
\text { for the management of food } \\
\text { allergy (2003) }\end{array}$ & 10 \\
\hline \multirow[t]{2}{*}{$\begin{array}{l}\text { Cydulka } \\
\text { (2003) } \\
\text { USA/Canada }\end{array}$} & \multirow[t]{2}{*}{$\begin{array}{l}\text { Prospective, } \\
\text { cohort }\end{array}$} & \multirow[t]{2}{*}{$\begin{array}{l}\text { Medical } \\
\text { record } \\
\text { review } \\
\text { Telephone } \\
\text { interviews }\end{array}$} & \multirow[t]{2}{*}{$\begin{array}{l}\text { Multicenter: } \\
29 \text { EDs }\end{array}$} & \multirow[t]{2}{*}{ Not specified } & \multirow[t]{2}{*}{$\begin{array}{l}397 \text { patients with } \\
\text { exacerbation COPD }\end{array}$} & $\begin{array}{l}\text { American thoracic society } \\
\text { standards for the diagnosis and } \\
\text { care of patients with chronic } \\
\text { obstructive pulmonary disease } \\
\text { (COPD) and asthma (1987) }\end{array}$ & \multirow[t]{2}{*}{10} \\
\hline & & & & & & $\begin{array}{l}\text { British Thoracic Society } \\
\text { guidelines for the management } \\
\text { of chronic obstructive pulmonary }\end{array}$ & \\
\hline $\begin{array}{l}\text { De Miguel-Yanes } \\
\text { (2006) } \\
\text { Spain }\end{array}$ & $\begin{array}{l}\text { Retrospective, } \\
\text { cohort }\end{array}$ & $\begin{array}{l}\text { Medical } \\
\text { record } \\
\text { review }\end{array}$ & $\begin{array}{l}\text { Monocenter: } \\
1 \text { ED }\end{array}$ & Not specified & $\begin{array}{l}53 \text { patients with } \\
\text { suspected sepsis }\end{array}$ & $\begin{array}{l}\text { Surviving sepsis campaign } \\
\text { guidelines for management of } \\
\text { severe sepsis and septic shock } \\
\text { (2004) }\end{array}$ & 9.5 \\
\hline $\begin{array}{l}\text { Doherty } \\
\text { (2007) } \\
\text { Australia }\end{array}$ & $\begin{array}{l}\text { Retrospective, } \\
\text { pre-test post- } \\
\text { test }\end{array}$ & $\begin{array}{l}\text { Database } \\
\text { review }\end{array}$ & $\begin{array}{l}\text { Multicenter: } 2 \\
\text { EDs }\end{array}$ & Not specified & $\begin{array}{l}215 \text { patients with } \\
\text { asthma }\end{array}$ & $\begin{array}{l}\text { NSW Department of Health } \\
\text { guideline for the optimal } \\
\text { treatment of chronic respiratory } \\
\text { diseases (2003) }\end{array}$ & 10 \\
\hline $\begin{array}{l}\text { Elkharrat } \\
\text { (1999) } \\
\text { France }\end{array}$ & $\begin{array}{l}\text { Prospective, } \\
\text { pre-test post } \\
\text { test }\end{array}$ & $\begin{array}{l}\text { Data } \\
\text { collection } \\
\text { chart }\end{array}$ & $\begin{array}{l}\text { Monocenter: } \\
1 \mathrm{ED}\end{array}$ & Not specified & $\begin{array}{l}389 \text { patients with } \\
\text { open wounds }\end{array}$ & $\begin{array}{l}\text { World Health Organisation } \\
\text { guideline for antitetanus } \\
\text { prophylaxis (1992) }\end{array}$ & 10 \\
\hline $\begin{array}{l}\text { Ferguson } \\
(2012) \\
\text { USA }\end{array}$ & $\begin{array}{l}\text { Retrospective, } \\
\text { cohort }\end{array}$ & $\begin{array}{l}\text { Medical } \\
\text { record } \\
\text { review }\end{array}$ & $\begin{array}{l}\text { Monocenter: } \\
1 \mathrm{ED}\end{array}$ & $\begin{array}{l}\text { Pediatric } \\
\text { emergency } \\
\text { physicians }\end{array}$ & $\begin{array}{l}167 \text { children with } \\
\text { fever }\end{array}$ & $\begin{array}{l}\text { Agency for Health Care Policy } \\
\text { and Research guideline for the } \\
\text { management of infants and } \\
\text { children } 0 \text { to } 36 \text { months of age } \\
\text { with fever without source (1993) }\end{array}$ & 9.5 \\
\hline $\begin{array}{l}\text { Grant } \\
(2006) \\
\text { UK }\end{array}$ & $\begin{array}{l}\text { Retrospective, } \\
\text { descriptive }\end{array}$ & $\begin{array}{l}\text { Medical } \\
\text { record } \\
\text { review }\end{array}$ & $\begin{array}{l}\text { Monocenter: } \\
1 \mathrm{ED}\end{array}$ & Not specified & $\begin{array}{l}473 \text { patients with } \\
\text { acute pain }\end{array}$ & $\begin{array}{l}\text { British Association of Accident } \\
\text { and Emergency Medicine } \\
\text { guideline for the management of } \\
\text { pain in adults (2005) }\end{array}$ & 10 \\
\hline $\begin{array}{l}\text { Jain } \\
\text { (2002) } \\
\text { USA }\end{array}$ & $\begin{array}{l}\text { Retrospective, } \\
\text { descriptive }\end{array}$ & $\begin{array}{l}\text { Medical } \\
\text { record } \\
\text { review }\end{array}$ & $\begin{array}{l}\text { Monocenter: } \\
1 \mathrm{ED}\end{array}$ & $\begin{array}{l}\text { Pediatric } \\
\text { residents } \\
\text { Fellows } \\
\text { Nurse } \\
\text { practitioners }\end{array}$ & $\begin{array}{l}229 \text { children with } \\
\text { fever }\end{array}$ & $\begin{array}{l}\text { Agency for Health Care Policy } \\
\text { and Research guideline for the } \\
\text { management of infants and } \\
\text { children } 0 \text { to } 36 \text { months of age } \\
\text { with fever without source (1993) }\end{array}$ & 9.5 \\
\hline $\begin{array}{l}\text { Kelly } \\
(2013) \\
\text { Australia }\end{array}$ & $\begin{array}{l}\text { Prospective, } \\
\text { descriptive }\end{array}$ & $\begin{array}{l}\text { Data } \\
\text { collection } \\
\text { chart }\end{array}$ & $\begin{array}{l}\text { Multicenter: } \\
38 \text { EDs }\end{array}$ & Not specified & $\begin{array}{l}1340 \text { patients with } \\
\text { acute asthma }\end{array}$ & $\begin{array}{l}\text { National Asthma Campaign } \\
\text { asthma management guideline } \\
\text { (1998) }\end{array}$ & 9.5 \\
\hline \multirow[t]{3}{*}{$\begin{array}{l}\text { Lee } \\
\text { (2001) } \\
\text { Taiwan }\end{array}$} & \multirow[t]{3}{*}{$\begin{array}{l}\text { Retrospective, } \\
\text { cohort }\end{array}$} & \multirow[t]{3}{*}{$\begin{array}{l}\text { Medical } \\
\text { record } \\
\text { review }\end{array}$} & \multirow[t]{3}{*}{$\begin{array}{l}\text { Multicenter: } 6 \\
\text { EDs }\end{array}$} & \multirow[t]{3}{*}{$\begin{array}{l}\text { Emergency } \\
\text { physicians }\end{array}$} & \multirow[t]{3}{*}{$\begin{array}{l}120 \text { patients with } \\
\text { acute asthma }\end{array}$} & $\begin{array}{l}\text { 1. British Thotacic Society } \\
\text { guidelines I \& II for the } \\
\text { management of asthma in adults } \\
\text { (1990\&1993) }\end{array}$ & \multirow[t]{3}{*}{9} \\
\hline & & & & & & $\begin{array}{l}\text { 2. National Heart, Lung and } \\
\text { Blood Institute guideline for the } \\
\text { diagnosis and management of } \\
\text { asthma (1991 \& } 1994 \text { \& 1997) }\end{array}$ & \\
\hline & & & & & & $\begin{array}{l}\text { 3. Asthma management } \\
\text { guidelines and therapeutic Issues } \\
\text { relating to the treatment of } \\
\text { asthma. Chest (1999) }\end{array}$ & \\
\hline $\begin{array}{l}\text { Mansbach } \\
\text { (2007) } \\
\text { USA }\end{array}$ & $\begin{array}{l}\text { Prospective, } \\
\text { cohort }\end{array}$ & $\begin{array}{l}\text { Medical } \\
\text { record } \\
\text { review } \\
\text { Interviews }\end{array}$ & $\begin{array}{l}\text { Multicenter: } \\
17 \text { EDs }\end{array}$ & Not specified & $\begin{array}{l}624 \text { children with } \\
\text { bronchiolitis }\end{array}$ & $\begin{array}{l}\text { American Academy of Pediatrics } \\
\text { Committee on Infectious } \\
\text { Diseases and Committee of Fetus } \\
\text { and Newborn guidelines for } \\
\text { prevention of respiratory }\end{array}$ & 9 \\
\hline
\end{tabular}


Table 2 Characteristics of included studies $(\mathbf{n}=\mathbf{3 5})$ (Continued)

$\begin{array}{lllll}\begin{array}{l}\text { Milks } \\ \text { (1999) } \\ \text { USA }\end{array} & \begin{array}{l}\text { Retrospective, } \\ \text { descriptive }\end{array} & \begin{array}{l}\text { Medical } \\ \text { record } \\ \text { review }\end{array} & \begin{array}{l}\text { Monocenter: } \\ 1 \mathrm{ED}\end{array} & \text { Not specified } \\ \begin{array}{l}\text { Muayqil } \\ \text { (2007) }\end{array} & \begin{array}{l}\text { Retrospective, } \\ \text { descriptive }\end{array} & \begin{array}{l}\text { Medical } \\ \text { record } \\ \text { ranada }\end{array} & \begin{array}{l}\text { Monocenter: } \\ 1 \text { ED }\end{array} & \begin{array}{l}\text { Emergency } \\ \text { physicians }\end{array} \\ \begin{array}{l}\text { Musacchio } \\ \text { (2009) }\end{array} & \begin{array}{l}\text { Retrospective, } \\ \text { descriptive }\end{array} & \begin{array}{l}\text { Medical } \\ \text { record }\end{array} & \begin{array}{l}\text { Monocenter: } \\ \text { (ED }\end{array} & \text { Not specified } \\ \text { USA } & & \text { review } & & \end{array}$

\begin{tabular}{|c|c|c|c|}
\hline $\begin{array}{l}\text { Pham } \\
(2007)\end{array}$ & $\begin{array}{l}\text { Cross } \\
\text { sectional, }\end{array}$ & $\begin{array}{l}\text { Database } \\
\text { review }\end{array}$ & $\begin{array}{l}\text { Multicenter: } \\
544 \text { EDs }\end{array}$ \\
\hline
\end{tabular}

descriptive

\section{Reid}

(2000)

Canada

$\begin{array}{lllll}\text { Roy } & \text { Prospective, } & \text { Data } & \text { Multicenter: } & \text { Emergency } \\ \text { (2006) } & \text { cohort } & \begin{array}{l}\text { collection } \\ \text { chart }\end{array} & 117 \mathrm{EDs} & \text { physicians } \\ \text { France \& Belgium } & & & & \end{array}$

\section{Salmeron}

(2001)

France

Shaked
(2009)
USA

Teismann

(2009)

USA
Retrospective, Medical descriptive

record

review

EDs

ulticenter: 3 Emergency

physicians

chart
Prospective,

cohort
Data
collection chart

Multicenter: 37 EDs

Emergency physicians

Retrospective, descriptive

Medical

record

review

\section{Monocenter: Not specified} $1 \mathrm{E}$

Retrospective, descriptive

Medical

record

review
Monocenter: $1 \mathrm{ED}$
Emergency residents Physician assistants syncytial virus infections:

indications for the use of

palivizumab and update on the use of RSV-IGIV (1998)

181 patients with asthma

45 patients with convulsive status epilepticus

163 patients with urinary tract infections, urinary symptoms or sexually transmitted disease

1492 patients with acute myocardial infarction

3955 patients with pneumonia

130 patients with asthma

Emergency nurses

1529 patients with suspected pulmonary embolism

4087 patients with 1. National Asthma Education and acute asthma Prevention Program guidelines for

56 children with

553 patients with suspected venous thromboembolism
National Heart, Lung and Blood Institute guideline for the diagnosis and management of asthma (1991)

Epilepsy Foundation of America guidelines for the management convulsive status epilepticus (1993)

Center for Disease Control and 9

Prevention: guideline for treatment of sexually transmitted diseases (2006)

Center for Medicare and Medicaid Services. Specification manual for national hospital quality measures for acute myocardial infarction and asthma (2007)

National guideline for the emergency management of asthma in adults (1996)

American College of Emergency Physicians Clinical Policies Committee. Clinical policy: critical issues in the evaluation and management of adult patients presenting with suspected pulmonary embolism (2003)

British Thoracic Society guidelines for the management of suspected acute pulmonary embolism (2003)

European Society of Cardiology Guidelines on diagnosis and management of acute pulmonary embolism (2000) the diagnosis and the management of asthma (1997)

2. British guidelines on asthma management, 1995 review and position statement (1997)

American Academy of Pediatrics (AAP) Practice Parameter: the neurodiagnostic evaluation of the child with a first simple febrile seizure (1996)

American College of Emergency Physicians Clinical Policies

Subcommittee on Suspected Pulmonary Embolism, evaluation and management of adult patients presenting with suspected pulmonary embolism (2003) 
Table 2 Characteristics of included studies $(\mathbf{n}=35)$ (Continued)

\begin{tabular}{|c|c|c|c|c|c|c|c|}
\hline $\begin{array}{l}\text { Thakore } \\
\text { (1999) } \\
\text { Scotland }\end{array}$ & $\begin{array}{l}\text { Retrospective, } \\
\text { descriptive }\end{array}$ & $\begin{array}{l}\text { Medical } \\
\text { record } \\
\text { review }\end{array}$ & $\begin{array}{l}\text { Monocenter: } \\
1 \text { ED }\end{array}$ & Not specified & $\begin{array}{l}100 \text { patients with } \\
\text { syncope }\end{array}$ & $\begin{array}{l}\text { American college of physicians } \\
\text { guideline for management of } \\
\text { patients with syncope (1997) }\end{array}$ & 9 \\
\hline $\begin{array}{l}\text { Trzeciak } \\
\text { (2006) } \\
\text { USA }\end{array}$ & $\begin{array}{l}\text { Retrospective, } \\
\text { cohort }\end{array}$ & $\begin{array}{l}\text { Medical } \\
\text { record } \\
\text { review }\end{array}$ & $\begin{array}{l}\text { Monocenter: } \\
1 \text { ED }\end{array}$ & $\begin{array}{l}\text { Emergency } \\
\text { physicians }\end{array}$ & $\begin{array}{l}22 \text { patients with } \\
\text { confirmed or } \\
\text { suspected sepsis }\end{array}$ & $\begin{array}{l}\text { Surviving sepsis campaign } \\
\text { guidelines for management of } \\
\text { severe sepsis and septic shock } \\
(2004)\end{array}$ & 10 \\
\hline \multirow[t]{3}{*}{$\begin{array}{l}\text { Tsai } \\
\text { (2009) } \\
\text { USA }\end{array}$} & $\begin{array}{l}\text { Retrospective, } \\
\text { cohort }\end{array}$ & $\begin{array}{l}\text { Medical } \\
\text { record } \\
\text { review }\end{array}$ & $\begin{array}{l}\text { Multicenter: } \\
2 \text { EDs }\end{array}$ & $\begin{array}{l}\text { Emergency } \\
\text { physicians }\end{array}$ & $\begin{array}{l}272 \text { patients with } \\
\text { COPD }\end{array}$ & $\begin{array}{l}\text { Global Initiative for Chronic } \\
\text { Obstructive Lung Disease } \\
\text { guidelines for the diagnosis, } \\
\text { management, and prevention of } \\
\text { chronic obstructive pulmonary } \\
\text { disease (2001) }\end{array}$ & 10 \\
\hline & & Interview & & & & $\begin{array}{l}\text { American College of Physicians } \\
\text { guidelines for Management of } \\
\text { acute exacerbations of chronic } \\
\text { obstructive pulmonary disease } \\
\text { (2001) }\end{array}$ & \\
\hline & & & & & & $\begin{array}{l}\text { American Thoracic Society and } \\
\text { European Respiratory Society } \\
\text { joint guidelines Standards for the } \\
\text { diagnosis and treatment of } \\
\text { patients with COPD (2004) }\end{array}$ & \\
\hline $\begin{array}{l}\text { Wright } \\
\text { (1998) } \\
\text { USA }\end{array}$ & $\begin{array}{l}\text { Retrospective, } \\
\text { descriptive }\end{array}$ & $\begin{array}{l}\text { Medical } \\
\text { record } \\
\text { review }\end{array}$ & $\begin{array}{l}\text { Monocenter: } \\
1 \text { ED }\end{array}$ & $\begin{array}{l}\text { Emergency } \\
\text { physicians }\end{array}$ & $\begin{array}{l}244 \text { patients who } \\
\text { received } \\
\text { vancomycin }\end{array}$ & $\begin{array}{l}\text { Center for Disease Control and } \\
\text { Prevention: Recommendations for } \\
\text { preventing the spread of } \\
\text { vancomycin resistance: } \\
\text { Recommendations of the Hospital } \\
\text { Infection Control Practices Advisory } \\
\text { Committee (1995) }\end{array}$ & 10 \\
\hline
\end{tabular}

Abbreviations: CICU Cardiac Intensive Care Unit, ED Emergency Department, EMS Emergency Medical Service, HEMS Helicopter Emergency Medical Service, MICU Mobile Intensive Care Unit, UH University Hospital.

Quality: assessed on a scale from 0 (poor quality) to 10 (excellent quality).

emergency medical technicians (EMT), and helicopter emergency medical service (HEMS) paramedics. Four studies were monocentric and six were multicentric. Seven studies were conducted in Europe and the remaining three in North America.

From the ten articles, a total of 40 recommendations were extracted. Four (10\%) were monitoring recommendations and 36 (90\%) were treatment recommendations. On these 40 recommendations, a total of 12 median adherence percentages were extracted or calculated, of which 2 (17\%) were monitoring percentages, and 10 (83\%) were treatment percentages. The distribution of the percentages across the different medical conditions and types of recommendations is displayed in Additional file 1: Figure 2.

Figure 2 shows median adherence percentages in the prehospital setting varying from $7.8 \%$ to $95 \%$. The three lowest median adherence percentages (7.8\%, 22\%, 27.5\%) came with cardiology treatment recommendations related to myocardial infarction [18] and cardiac arrest [27,28], whereas the three highest median adherence percentages $(77.5 \%, 79.8 \%, 95 \%)$ came with treatment recommendations related to oxygen administration [29] and septicaemia [25], and to one monitoring recommendation related to oxygen administration [29]. Looking at medical functions, monitoring recommendations came with less variation in adherence when compared to the treatment recommendations, and monitoring recommendations came with higher median adherence percentages. Regarding the medical conditions, cardiology treatment recommendations are less often adhered to than treatment recommendations for other medical conditions.

\section{Emergency department setting}

Twenty-six studies describing adherence to (inter)national guidelines in the ED setting were identified. These guidelines covered cardiology [18,34], pulmonology [19,20,23,34-40], neurology [21,24], infectious diseases [4147 , and 'other' conditions [22,48-52] (Table 3). Professionals were (paediatric) emergency physicians, medical fellows, emergency nurses, and nurse practitioners. Fourteen studies were monocentric and twelve were multicentric. Sixteen studies were conducted in North America, seven in Europe, two in Australia, and one in Asia.

From the 26 studies, a total of 161 recommendations were extracted. Fifty-one (32\%) were diagnostic recommendations, one $(<1 \%)$ was a monitoring recommendation, $102(63 \%)$ were treatment recommendations, and seven (4\%) were organisational recommendations. On these 161 
Table 3 Guideline topics

\section{Medical condition}

Cardiology

Pulmonology

Infectious diseases

Other

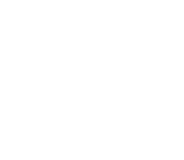

\section{Prehospital setting}

Cardiac arrest [26-28]

Myocardial infarction [18]

Sedation [32]

Traumatic brain injury [30-33]

Oxygen administration [29]

Meningococcal septicaemia [25]

\section{Emergency department setting}

Myocardial infarction [18,34]

Convulsive status epilepticus [21]

Syncope [24]

Bronchiolitis [37]

Asthma [20,23,35,36,38,39]

COPD $[19,40]$

Pneumonia [34]

Antibiotic therapy [47]

Antitetanus prophylaxis [43]

Fever [42]

Febrile seizures $[44,45]$

Sepsis $[41,46]$

Allergic reactions to food [49]

Antithrombotic therapy [48]

Pain [50]

Pulmonary and venous embolisms [22,52]

Urinary complaints/sexually transmitted diseases [51]

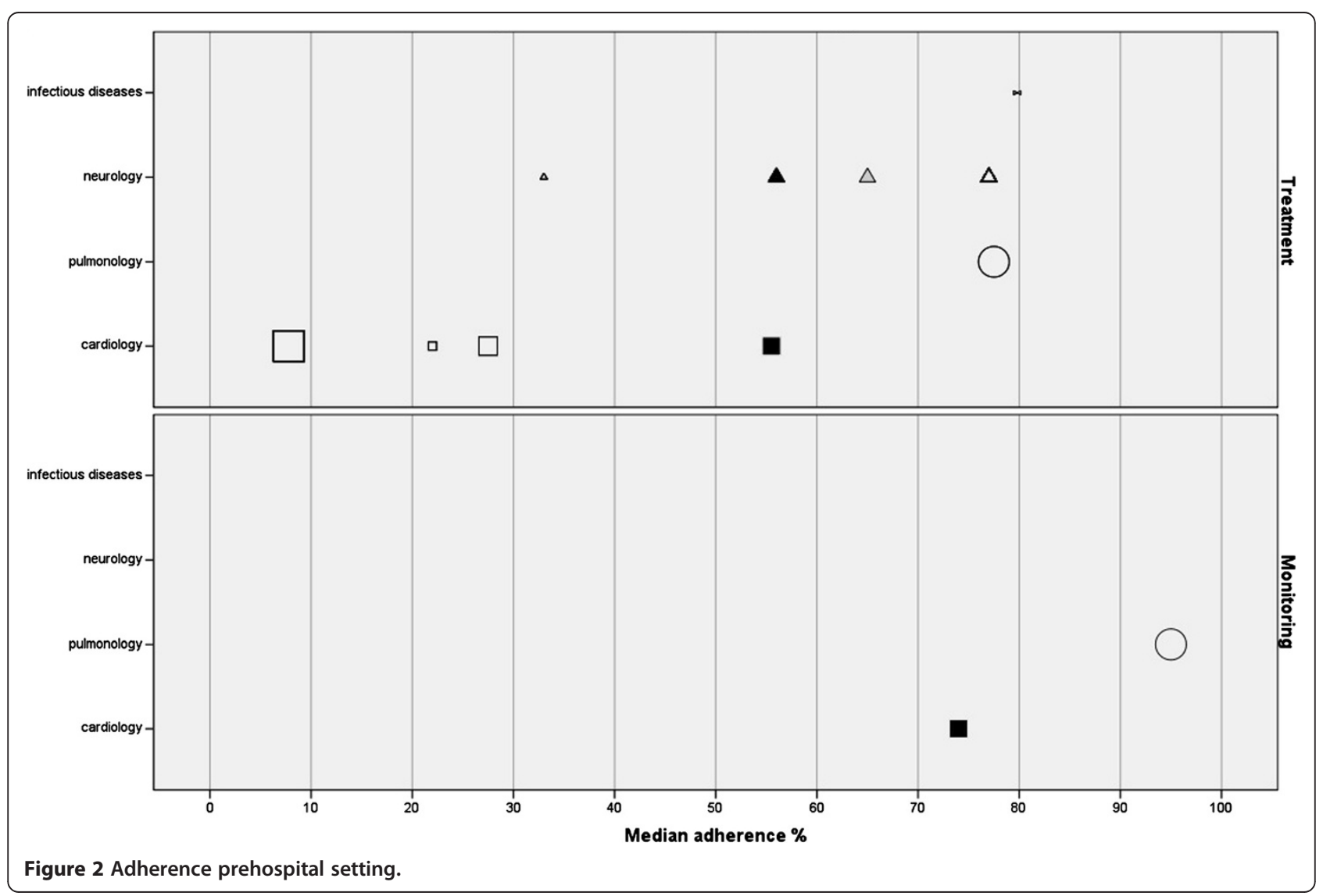


recommendations, a total of 40 median adherence percentages were extracted or calculated. Fourteen (35\%) were percentages on the uptake of recommendations for diagnostics, one $(2.5 \%)$ was a percentage for adherence to a recommendation on monitoring, 20 (50\%) were percentages for the uptake of treatment recommendations, and five (12.5\%) were adherence percentages for organisational recommendations. The distribution of the percentages across the different medical conditions and types of recommendations is displayed in Additional file 2: Figure 3.

Figure 3 shows a wide variation in adherence percentages in the ED setting, varying from $0 \%$ to $98 \%$. The three lowest median adherence percentages $(0 \%, 7.8 \%$, $12.5 \%$ ) came with a monitoring recommendation related to sepsis [41], a treatment recommendation related to myocardial infarction [18], and a diagnostic recommendation related to asthma [20]. The highest median adherence percentages $(88.5 \%, 91 \%, 98 \%)$ came with a diagnostic recommendation related to COPD [40], and treatment recommendations related to asthma [38] and sepsis [46]. Looking at medical functions, diagnostic and organisational recommendations came with higher median adherence percentages compared to the treatment recommendations. Among medical conditions, pulmonary treatment recommendations came with higher median adherence percentages, and cardiology treatment recommendations came with lower median adherence percentages compared to other conditions.

\section{Influencing factors}

Eight studies reported factors influencing adherence $[18,20,22,26,34,37,42,51]$. These factors were related to the

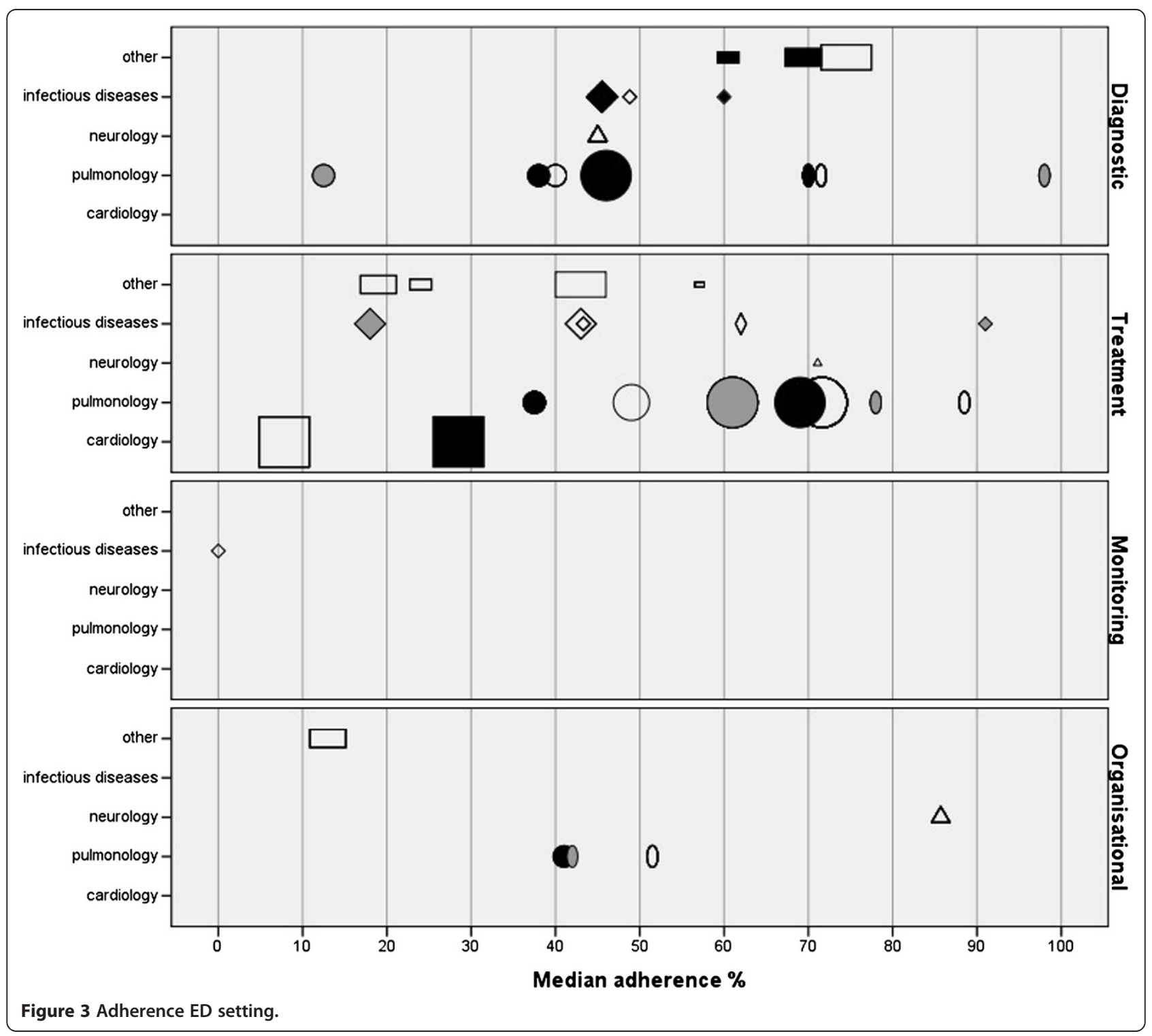


Table 4 Influencing factors

\begin{tabular}{lll}
\hline Domain & $\begin{array}{l}\text { Influencing } \\
\text { factor }\end{array}$ & Medical condition \\
\hline $\begin{array}{l}\text { Patient } \\
\text { characteristics }\end{array}$ & Age & Cardiology \\
& Patients with ST-segment elevation myocardial infarction aged $\leq 75$ years were more likely to receive care in \\
accordance with the guideline [18] \\
- Patients with acute myocardial infarction aged $<55$ years were more likely to receive aspirin [34] \\
Pulmonology \\
- Patients with pneumonia aged $<18$ years were more likely to receive recommended antibiotics [34] \\
- Patients with pneumonia aged $<18$ years were less likely to be monitored with pulse oximetry [34] \\
- Patients with suspected pulmonary embolism aged $>75$ years were less likely to be diagnosed in accordance \\
with the guideline [22]
\end{tabular}
with the guideline [22]

- Children with bronchiolitis whose gestational age was 30 weeks were more likely to receive palivizumab compared to children whose gestational age was 32 weeks [37]

Other

- Patients with urinary complaints aged >19 years were more likely to be taken their sexual history [51]

- Children with fever who were aged 28-59 days were more likely to receive complete blood cell count, blood culture, urine culture, cerebrospinal fluid culture and viral studies compared to children who were aged 60-90 days [42]

Gender Cardiology

- Male patients with acute myocardial infarction were more likely to receive B-blockers [34]

- Male patients with cardiac arrest were more likely to receive treatment in accordance with the guidelines [26]

Weight Pulmonology

- Children with bronchiolitis with birth-weight <3lbs were more likely to receive palivizumab [37]

Current disease/ Cardiology

condition

- Patients with ST-segment elevation myocardial infarction with a symptom onset 08.00-20.00 were more likely to receive care in accordance with the guideline than patients with a symptom onset 20.00-08.00 [18]

- Patients with ST-segment elevation myocardial infarction with a typical STEMI on the ECG were more likely to receive care in accordance with the guideline compared to patients without a typical STEMI on the ECG [18]

- Patients with cardiac arrest of whom the arrest was witnessed or with an initial rhythm of VFNT were more likely to receive care in accordance with the guideline than patients with an unwitnessed arrest of initial rhythm other than VFNT [26]

- Patients with cardiac arrest with a longer time interval between return of spontaneous circulation and hospital admission were more likely to receive care in accordance with the guideline compared to patients with a shorter time interval [26]

Pulmonology

- Patients with suspected pulmonary embolism currently receiving anticoagulation were less likely to be diagnosed in accordance with the guideline compared to patients with anticoagulation [22]

- Children with bronchiolitis with a history of wheezing were more likely to receive palivizumab than patients without a history of wheezing [37]

Other

- Patients with urinary complaints with a history of fever were more likely to be taken their sexual history than patients without a history of fever [51]

- Patients with urinary complaints with genital discharge were more likely to be taken their sexual history than patients without genital discharge [51]

Race Cardiology

- Patients with acute myocardial infarction of Hispanic race were less likely to receive aspirin compared to patients of white or nonwhite race [34]

Pulmonology

- Patients with pneumonia of nonwhite race were less likely to be monitored with pulse oximetry compared to patients of white or hispanic race [34] 


\begin{tabular}{|c|c|c|}
\hline & & $\begin{array}{l}\text { - Patients with acute myocardial infarction with a private insurance were more likely to receive aspirin than } \\
\text { patients with a medicare or Medicaid insurance [34] }\end{array}$ \\
\hline & & Pulmonology \\
\hline & & $\begin{array}{l}\text { - Patients with pneumonia with a private insurance were more likely to receive antibiotics than patients with a } \\
\text { medicare of Medicaid insurance [34] }\end{array}$ \\
\hline & Comorbidity & Cardiology \\
\hline & & $\begin{array}{l}\text { - Patients with cardiac arrest with a prior neurological disease were less likely to receive care in accordance } \\
\text { with the guideline compared to patients without prior neurological disease [26] }\end{array}$ \\
\hline & & Pulmonology \\
\hline & & $\begin{array}{l}\text { - Patients with suspected pulmonary embolism with known heart failure, known chronic lung disease or } \\
\text { current/recent pregnancy were less likely to be diagnosed in accordance with the guideline than patients } \\
\text { without known heart failure, chronic lung disease or current/recent pregnancy [22] }\end{array}$ \\
\hline & & $\begin{array}{l}\text { - Patients with suspected pulmonary embolism with previous thromboembolism were more likely to be } \\
\text { diagnosed in accordance with the guideline than patients without previous thromboembolism [22] }\end{array}$ \\
\hline & Time of & Other \\
\hline & presentation & $\begin{array}{l}\text { - Patients with urinary complaints who presented in the evening were more likely to be taken their sexual } \\
\text { history compared to patients who presented in over daytime [51] }\end{array}$ \\
\hline Organisational & Location & Cardiology \\
\hline & & $\begin{array}{l}\text { - Patients with ST-segment elevation myocardial infarction treated in an urban ED were more likely to be } \\
\text { treated in accordance with the guideline compared to patients treated in a rural ED [18] }\end{array}$ \\
\hline & & $\begin{array}{l}\text { - Patients with acute myocardial infarction treated in a Midwest or Southern ED were less likely to receive ß- } \\
\text { blockers compared to patients treated in a northeast or west ED [34] }\end{array}$ \\
\hline & & Pulmonology \\
\hline & & $\begin{array}{l}\text { - Patients with pneumonia treated in a Southern ED are less likely to receive antibiotics compared to patient } \\
\text { treated in a northeast, west or midwest ED [34] }\end{array}$ \\
\hline & & $\begin{array}{l}\text { - Patients with pneumonia treated in a metropolitan ED are more likely to receive antibiotics and are more } \\
\text { likely to be monitored with pulse oximetry compared to patients in a non-metropolitan ED [34] }\end{array}$ \\
\hline & & $\begin{array}{l}\text { - Patients with asthma treated in medical centres were more likely to be diagnosed with oximetry or arterial } \\
\text { blood gas compared to patients in regional and district EDs [20] }\end{array}$ \\
\hline & Presence of a & Cardiology \\
\hline & physıcian & $\begin{array}{l}\text { - Patients with cardiac arrest where a prehospital physician was present on scene were more likely to receive } \\
\text { care in accordance with the guideline than patients without prehospital physician presence [26] }\end{array}$ \\
\hline & Ownership of the & Cardiology \\
\hline & & $\begin{array}{l}\text { - Patients with acute myocardial infarction treated in an ED with governmental or non-federal ownership are } \\
\text { less likely to receive aspirin than patients treated in an nonprofit or proprietary ED [34] }\end{array}$ \\
\hline & & Pulmonology \\
\hline & & $\begin{array}{l}\text { - Patients with pneumonia treated in an ED with governmental or non-federal ownership are less likely to } \\
\text { receive antibiotics compared to patients treated in an nonprofit or proprietary ED [34] }\end{array}$ \\
\hline
\end{tabular}

patient (age, race, sex, weight, time of presentation, insurance status, current disease/condition and comorbidity) and to the organisation (presence of an emergency physician, ownership (non-federal or governmental) hospital/ ED and location) (Table 4). When categorised along medical conditions, the patient related influencing factors had different directions and no clear patterns existed, although male sex, lower age and a disease specific condition (rhythm on the electrocardiogram) seemed to positively influence adherence to cardiology guidelines. As for organisational factors, there seemed to be a pattern that treatment in a governmental or non-federal ED negatively influences adherence to (inter)national guidelines.

\section{Discussion}

This systematic review aimed to give an overview of professionals' adherence to (inter)national guidelines and protocols in the emergency medical dispatch, prehospital ambulance and ED settings. In addition, factors influencing adherence were explored. Thirty-five articles describing adherence to (inter)national prehospital and ED guidelines were identified. No studies describing adherence to protocols or studies in the emergency medical dispatch setting were identified. Despite the life-threatening and urgent conditions covered by the guidelines, results showed a wide variation in adherence. Extracted factors influencing adherence were related to the patient and to the organisation. 
For both the prehospital and ED setting adherence showed a wide variation. Suboptimal adherence has also been shown in other critical care fields, such as the intensive care unit $[53,54]$ and the recovery room $[55,56]$, but also on more general topics as hand hygiene [57] and medication safety [58]. It is possible that the wide variation in adherence is due to often poor evidence-based prehospital guidelines [59], to differences in guideline quality or due to justified deviations as guidelines have to be tailored to unique patients. Unjustified deviations may also contribute to this wide variation in adherence, as situations where guideline deviations are desired are unclear [60]. Specifically regarding the ED setting, another reason for suboptimal adherence may be that guidance for some ED presentations are derived from guidelines of specialties outside the ED as ED guidelines are lacking. As guideline development programmes increasingly become evidence based [61] and guidelines represent the standard of care, our results probably also imply that many patients in the prehospital and ED setting do not receive appropriate care.

Guideline recommendations were extracted to categorise the adherence percentages into recommendation categories in relation to medical function and medical condition. For medical function in the prehospital setting, monitoring recommendations came with higher adherence percentages compared to treatment recommendations. In the ED setting, diagnostic and organisational recommendations came with higher median adherence percentages compared to treatment recommendations. This may indicate that the type of medical function influences adherence to (inter) national guideline recommendations. This result is supported by a previous non-emergency care review, which showed that characteristics of the guideline recommendations (medical condition, type of procedure, complexity) influence guideline adherence [17]. A possible explanation for the large variation in adherence rates for different types of guideline recommendations may be the existence of barriers specifically for individual recommendations rather than guidelines as a whole [5]. For instance, the strength of evidence and the impact on patient outcomes may vary across individual recommendations. Another explanation may be that guidelines contain too many recommendations to adhere to, or that EMSs and EDs are not able to implement all recommendations at the same time and make choices. If this is the case, guidelines could be translated into more efficient, practical and feasible protocols, algorithms, and decision trees.

In addition to differences for types of medical functions of guideline recommendations, variation in adherence percentages for medical conditions was observed. This variation has been reported previously [17]. Especially the cardiology and 'other' guidelines came with lower adherence percentages compared to other medical conditions. These cardiology guidelines cover cardiac arrest and ST- elevation myocardial infarction, two conditions known for their high mortality rates $[62,63]$, while pain ('other' guideline) is reported to be the main complaint for patients to use emergency care [64].

Factors influencing adherence were reported in eight studies [18,20,22,26,34,37,42,51]. These factors can be clustered into factors related to the patient and to the organisation. No professional related factors were studied, which is remarkable as previous studies showed that individual experience, professional autonomy, attitudes and believes also determine to what degree professionals adhere to a guideline and that additional, individual training for ambulance nurses improves adherence to national prehospital protocols [65-67]. Additional research is needed, focussing on the perspectives of professionals, patients, organisations, social environment and characteristics of guidelines and protocols [7]. This knowledge can be used to develop and revise guidelines and protocols [68] and to tailor strategies to improve adherence. It is even argued that these strategies should be tailored to individual guideline recommendations instead of the guideline as a whole [5]. A systematic review showed that strategies tailored to identified barriers are effective to improve professional practice [69]. For the emergency care setting, previous studies showed that strategies tailored to influencing factors improve adherence to guidelines and protocols for patients with asthma, acute coronary syndromes and ST-elevation myocardial infarction $[35,70,71]$. To monitor adherence and assess effectiveness of implementation strategies it is recommended that guidelines contain clinical indicators [72]. These indicators have shown to be useful to assess and monitor guideline adherence [73]. Therefore, quality indicators should be part of the guideline development process or should be integrated in existing guidelines.

Besides implementations strategies, solid evidence based recommendations and a clear relationship between guideline adherence and patient outcomes may be the strongest motivators for emergency care professionals to adhere to guidelines. Generally, it is stated that especially prehospital care lacks strong evidence and clear indicators to measure effectiveness [74]. In this review, four studies assessed the relationship between adherence and patient outcomes. Three of these showed that adherence to guidelines improves patient outcomes by decreasing mortality and adverse events for patients with ST-segment elevation myocardial infarction, cardiac arrest and suspected pulmonary embolism $[18,22,26]$. However, the limited number of studies assessing the relationship prevents us from drawing firm conclusions. Therefore, future research should focus on the relationship between guideline adherence and patient outcomes.

We did not find studies in the emergency medical dispatch setting which met our inclusion criteria. Since the 
dispatch center is the first in the 'chain of emergency care', adherence to dispatch guidelines and protocols is important to correctly identify and prioritize the most urgent patients. Therefore, we recommend additional research on guideline and protocol adherence in this specific setting. One article assessed adherence in two consecutive emergency settings [18]. It is widely recognized that patients enter a 'chain of emergency care', and therefore assessment of adherence to guidelines and protocols in consecutive settings seems reasonable.

\section{Limitations of included studies}

The included studies predominantly had a retrospective design and used patient records or databases to retrieve their data. These methods incorporate a high risk of bias. The second problem we faced was the fact that the included studies incorporated a variability of guidelines, medical conditions, medical functions, designs, and methods, and that some studies assessed adherence to 'foreign' guidelines. Therefore, an overall comparison between the studies was difficult. Third, the included studies used several synonyms and definitions of adherence, including compliance, deviation, and 'guideline follow-up'. Literature shows no clear and widely used definition of adherence, while agreement on a useful definition would assist research. Finally, none of the included studies addressed the seriousness of the deviations, which may have been useful as previous research indicated that $45 \%$ of guideline deviations can be categorised as serious or very serious [68].

\section{Study limitations}

A limitation regards the assessment of reporting quality of the included articles, for which we used a checklist based on the STROBE and TREND statements. We are aware that the intended goal of these statements is to provide guidance on reporting research rather than assessing study quality, but adequate quality assessment tools for observational studies are lacking [75]. Furthermore, the differences in settings, personnel, disease processes, and guidelines made interpretation of the results exceedingly challenging.

\section{Conclusion}

Despite the often life-threatening and vital topics of the guidelines, adherence to (inter)national prehospital and ED guidelines showed a wide variation and ranges from $7.8-95 \%$ and $0-98 \%$ respectively. Research on adherence in the emergency medical dispatch setting is lacking. In the prehospital setting monitoring recommendations came with higher adherence percentages than treatment recommendations. For both settings, the cardiology treatment recommendations were less adhered to than recommendations for other medical conditions. These results indicate that the medical function and medical condition into which a guideline recommendation can be categorised might influence adherence. Further factors influencing adherence were related to the patient and the organisation. Factors related to professionals were not found. Further research should focus on identifying factors influencing adherence, taking into account the perspectives of the professional, patients, organisation, and characteristics of the guidelines. On the basis of these influencing factors, strategies can be developed to improve adherence to prehospital and ED guidelines, with the ultimate goal to ensure that patients receive appropriate care.

\section{Additional files}

Additional file 1: Legend Figure 2 prehospital setting.

Additional file 2: Legend Figure 3 ED setting.

\section{Competing interests}

The authors declare that they have no competing interests.

\section{Authors' contributions}

Study design (RE, LV, MV, JM, TVA). Data collection and analysis (RE, LV, MV, SM, JM, TVA). Quality assessment (RE, LV, JM, TVA). Manuscript preparation (RE, LV, SM, MV, JM, TVA). All authors read and approved the final manuscript.

\section{Author details}

${ }^{1}$ Research group for Acute Care, Faculty of Health and Social Studies, HAN University of Applied Sciences, Verlengde Groenestraat 75, Nijmegen 6525 EJ, The Netherlands. ${ }^{2}$ Canisius Wilhelmina Hospital, Weg door Jonkerbos 100 , Nijmegen 6532 SZ, The Netherlands. 'Sint Elisabeth Hospital, Hilvarenbeekseweg 60, Tilburg 5022 GC, The Netherlands. ${ }^{4}$ Scientific Institute for Quality of Healthcare, Radboud University Nijmegen Medical Centre, Geert Grooteplein 21, Nijmegen 6525 EZ, The Netherlands. ${ }^{5}$ Research group for Acute Care, Faculty of Health and Social Studies, HAN University of Applied Sciences, PO Box 6960, 6503 GL, Nijmegen, The Netherlands.

Received: 22 October 2012 Accepted: 29 January 2013

Published: 19 February 2013

\section{References}

1. Thomas L, Cullum N, McColl E, Rousseau N, Soutter J, Steen N: Guidelines in professions allied to medicine. Rev: Cochrane Database Syst; 2009.

2. Holleman G, Eliens A, Van Vliet M, Van Achterberg T: Promotion of evidence-based practice by professional nursing associations: literature review. J Adv Nurs 2006, 53:702-709.

3. Pines JM, Fee C, Fermann GJ, Ferroggiaro AA, Irvin CB, Mazer M, Frank PW, Schuur JD, Weber EJ, Pollack CV: The role of the society for academic emergency medicine in the development of guidelines and performance measures. Acad Emerg Med 2010, 17:130-140.

4. Field MJ: Clinical practice guidelines: Directions for a new program. Washington DC: National Academy Press; 1990.

5. Lugtenberg M, Burgers JS, Westert GP: Effects of evidence-based clinical practice guidelines on quality of care: a systematic review. Qual Saf Health Care 2009, 18:385-392.

6. Grol R: Improving Patient Care. The Implementation of Change in Clinical Practice. Toronto: Elsevier Science; 2005.

7. Van Achterberg T, Schoonhoven L, Grol R: Nursing implementation science: how evidence-based nursing requires evidence-based implementation. J Nurs Scholarsh 2008, 40:302-310.

8. Grol R, Grimshaw J: From best evidence to best practice: effective implementation of change in patients' care. Lancet 2003, 362:1225-1230

9. McGlynn EA, Asch SM, Adams J, Keesey J, Hicks J, DeCristofaro A, Kerr EA: The quality of health care delivered to adults in the United States. N Engl J Med 2003, 348:2635-2645 
10. Francke $A L$, Smit MC, de Veer AJ, Mistiaen P: Factors influencing the implementation of clinical guidelines for health care professionals: a systematic meta-review. BMC Med Inform Decis Mak 2008, 8:38-6947-8-38.

11. Cone DC: Knowledge translation in the emergency medical services: research agenda for advancing prehospital care. Acad Emerg Med 2007, 14:1052-1057.

12. Agrawal $P$, Kosowsky JM: Clinical practice guidelines in the emergency department. Emerg Med Clin North Am 2009, 27:555-567.

13. Moher D, Liberati A, Tetzlaff J, Altman DG: Preferred reporting items for systematic reviews and meta-analyses: the PRISMA statement. BMJ 2009 339:b2535.

14. Henry K, Campbell S, Maki M: A comparison of observed and selfreported compliance with universal precautions among emergency department personnel at a Minnesota public teaching hospital: implications for assessing infection control programs. Ann Emerg Med 1992, 21:940-946.

15. Von Elm E, Altman DG, Egger M, Pocock SJ, Gotzsche PC, Vandenbroucke JP, STROBE Initiative: The Strengthening the Reporting of Observational Studies in Epidemiology (STROBE) statement: guidelines for reporting observational studies. J Clin Epidemiol 2008, 61:344-349.

16. Des Jarlais DC, Lyles C, Crepaz N: Improving the reporting quality of nonrandomized evaluations of behavioral and public health interventions: the TREND statement. Am J Public Health 2004, 94:361-366.

17. Grilli R, Lomas J: Evaluating the message: the relationship between compliance rate and the subject of a practice guideline. Med Care 1994, 32:202-213.

18. Charpentier S, Sagnes-Raffy C, Cournot M, Cambou JP, Ducasse JL, Lauque $D$, Puel J: Determinants and prognostic impact of compliance with guidelines in reperfusion therapy for ST-segment elevation myocardial infarction: results from the ESTIM Midi-Pyrenees Area. Arch Cardiovasc Dis 2009, 102:387-396.

19. Cydulka RK, Rowe BH, Clark S, Emerman CL, Camargo CA: Emergency department management of acute exacerbations of chronic obstructive pulmonary disease in the elderly: the Multicenter Airway Research Collaboration. J Am Geriatr Soc 2003, 51:908-916.

20. Lee PL, Luo JP, Shieh WM, Nien CT, Yang PC, Kuo SH: Hospital-based management of acute asthmatic exacerbation: an assessment of physicians' behavior in Taiwan. J Asthma 2001, 38:575-583.

21. Muayqil T, Rowe BH, Ahmed SN: Treatment adherence and outcomes in the management of convulsive status epilepticus in the emergency room. Epileptic Disord 2007, 9:43-50.

22. Roy PM, Meyer G, Vielle B, Le GC, Verschuren F, Carpentier F, Leveau P, Furber A: Appropriateness of diagnostic management and outcomes of suspected pulmonary embolism. Ann Intern Med 2006, 144:157-164.

23. Salmeron S, Liard R, Elkharrat D, Muir J, Neukirch F, Ellrodt A: Asthma severity and adequacy of management in accident and emergency departments in France: a prospective study. Lancet 2001, 358:629-635.

24. Thakore SB, Crombie I, Johnston M: The management of syncope in a British emergency department compared to recent American guidelines. Scott Med J 1999, 44:155-157.

25. Cooke ME: Prehospital administration of benzyl penicillin by paramedics in the UK. JEPHC 2005, 3

26. Kirves H, Skrifvars MB, Vahakuopus M, Ekstrom K, Martikainen M, Castren M: Adherence to resuscitation guidelines during prehospital care of cardiac arrest patients. Eur J Emerg Med 2007, 14:75-81.

27. Scliopou J, Mader TJ, Durkin L, Stevens M: Paramedic compliance with ACLS epinephrine guidelines in out-of-hospital cardiac arrest Prehosp Emerg Care 2006, 10:394-396.

28. Wik L, Kramer-Johansen J, Myklebust H, Sorebo H, Svensson L, Fellows B, Steen PA: Quality of cardiopulmonary resuscitation during out-of -hospital cardiac arrest. JAMA 2005, 293:299-304.

29. Hale KE, Gavin C, O'Driscoll BR: Audit of oxygen use in emergency ambulances and in a hospital emergency department. Emerg Med J 2008, 25:773-776

30. Caulfield EV, Dutton RP, Floccare DJ, Stansbury LG, Scalea TM: Prehospital hypocapnia and poor outcome after severe traumatic brain injury. J Trauma 2009, 66:1577-1582.

31. Franschman G, Peerdeman SM, Greuters S, Vieveen J, Brinkman AC, Christiaans HM, Toor E, Jukema GN, Loer SA, Boer C: Prehospital endotracheal intubation in patients with severe traumatic brain injury: guidelines versus reality. Resuscitation 2009, 80:1147-1151.
32. Jeremie $N$, Seltzer $S$, Lenfant F, Ricard-Hibon A, Facon A, Cabrita B, Messant I, d'Athis P, Freysz M: Rapid sequence induction: a survey of practices in three French prehospital mobile emergency units. Eur J Emerg Med 2006, 13:148-155.

33. Thomas SH, Orf J, Wedel SK, Conn AK: Hyperventilation in traumatic brain injury patients: inconsistency between consensus guidelines and clinical practice. J Trauma 2002, 52:47-52.

34. Pham JC, Kelen GD, Pronovost PJ: National study on the quality of emergency department care in the treatment of acute myocardial infarction and pneumonia. Acad Emerg Med 2007, 14:856-863.

35. Doherty SR, Jones PD, Davis L, Ryan NJ, Treeve V: Evidence-based implementation of adult asthma guidelines in the emergency department: a controlled trial. Emerg Med Australas 2007, 19:31-38.

36. Kelly AM, Powell C, Kerr D: Snapshot of acute asthma: treatment and outcome of patients with acute asthma treated in Australian emergency departments. Intern Med J 2003, 33:406-413.

37. Mansbach J, Kunz S, Acholonu U, Clark S, Camargo CA: Evaluation of compliance with palivizumab recommendations in a multicenter study of young children presenting to the emergency department with bronchiolitis. Pediatr Emerg Care 2007, 23:362-367.

38. Milks CJ, Oppenheimer JJ, Bielory L: Comparison of emergency room asthma care to National Guidelines. Ann Allergy Asthma Immunol 1999, 83:208-211.

39. Reid J, Marciniuk DD, Cockcroft DW: Asthma management in the emergency department. Can Respir J 2000, 7:255-260.

40. Tsai CL, Ginde AA, Blanc PG, Camargo CA: Improved care of acute exacerbation of chronic obstructive pulmonary disease in two academic emergency departments. Int. J. Emerg. Med. 2009, 2:111-116.

41. De Miguel-Yanes JM, Andueza-Lillo JA, Gonzalez-Ramallo VJ, Pastor L, Munoz J: Failure to implement evidence-based clinical guidelines for sepsis at the ED. Am J Emerg Med 2006, 24:553-559.

42. Ferguson CC, Roosevelt G, Bajaj L: Practice patterns of pediatric emergency medicine physicians caring for young febrile infants. Clin. Pediatr. (Phila) 2010, 49:350-354.

43. Elkharrat D, Boyer CA, Raskine L, Durand-Zaleski I, Zerbani A, Caulin C, Chastang C: Impact of guidelines to alter antitetanus prophylaxis practices and reduce costs in the emergency department. Am J Ther 1999, 6:203-209.

44. Jain S, Sullivan K: Ceftriaxone use in the emergency department: are we doing it right? Pediatr Emerg Care 2002, 18:259-264.

45. Shaked O, Pena BM, Linares MY, Baker RL: Simple febrile seizures: are the AAP guidelines regarding lumbar puncture being followed? Pediatr Emerg Care 2009, 25:8-11.

46. Trzeciak S, Dellinger RP, Abate NL, Cowan RM, Stauss M, Kilgannon JH, Zanotti S, Parrillo JE: Translating research to clinical practice: a 1-year experience with implementing early goal-directed therapy for septic shock in the emergency department. Chest 2006, 129:225-232.

47. Wright SW, Wrenn KD: Appropriateness of vancomycin use in the emergency department. Ann Emerg Med 1998, 32:531-536.

48. Atreja A, El-Sameed YA, Jneid H, Hoogwerf BJ, Peacock WF: Elevated international normalized ratio in the ED: clinical course and physician adherence to the published recommendations. Am J Emerg Med 2005, 23:40-44.

49. Clark S, Bock SA, Gaeta TJ, Brenner BE, Cydulka RK, Camargo CA: Multicenter study of emergency department visits for food allergies. J Allergy Clin Immunol 2004, 113:347-352.

50. Grant PS: Analgesia delivery in the ED. Am J Emerg Med 2006, 24:806-809.

51. Musacchio NS, Gehani S, Garofalo R: Emergency department management of adolescents with urinary complaints: missed opportunities. J Adolesc Health 2009, 44:81-83.

52. Teismann NA, Cheung PT, Frazee B: Is the ordering of imaging for suspected venous thromboembolism consistent with D-dimer result? Ann Emerg Med 2009, 54:442-446.

53. Cahill NE, Heyland DK: Bridging the guideline-practice gap in critical care nutrition: a review of guideline implementation studies. JPEN J Parenter Enteral Nutr 2010, 34:653-659.

54. Kett DH, Cano E, Quartin AA, Mangino JE, Zervos MJ, Peyrani P, Cely CM, Ford $K D$, Scerpella EG, Ramirez JA: Implementation of guidelines for management of possible multidrug-resistant pneumonia in intensive care: an observational, multicentre cohort study. Lancet Infect Dis 2011, 11:181-189. 
55. Gustafsson UO, Hausel J, Thorell A, Ljungqvist O, Soop M, Nygren J: Adherence to the enhanced recovery after surgery protocol and outcomes after colorectal cancer surgery. Arch Surg 2011, 146:571-577.

56. Franck M, Radtke FM, Baumeyer A, Kranke P, Wernecke KD, Spies CD: Adherence to treatment guidelines for postoperative nausea and vomiting. How well does knowledge transfer result in improved clinical care? Anaesthesist 2010, 59:524-528.

57. Erasmus V, Daha TJ, Brug H, Richardus JH, Behrendt MD, Vos MC, van Beeck EF: Systematic review of studies on compliance with hand hygiene guidelines in hospital care. Infect Control Hosp Epidemiol 2010, 31:283-294.

58. Juarez A, Gacki-Smith J, Bauer MR, Jepsen D, Paparella S, VonGoerres B, MacLean S: Barriers to emergency departments' adherence to four medication safety-related Joint Commission National Patient Safety Goals. Jt Comm J Qual Patient Saf 2009, 35:49-59.

59. Fevang E, Lockey D, Thompson J, Lossius HM, Torpo Research Collaboration: The top five research priorities in physician-provided pre-hospital critical care: a consensus report from a European research collaboration. Scand J Trauma Resusc Emerg Med 2011, 19:57.

60. Koerselman GF, Korzec A: A proposed checklist when deviating from guidelines. Ned Tijdschr Geneeskd 2008, 152:1757-1759.

61. Burgers JS, Grol R, Klazinga NS, Makela M, Zaat J, AGREE Collaboration: Towards evidence-based clinical practice: an international survey of 18 clinical guideline programs. Int J Qual Health Care 2003, 15:31-45.

62. Nichol G, Thomas E, Callaway CW, Hedges J, Powell JL, Aufderheide TP, Rea T, Lowe R, Brown T, Dreyer J, Davis D, Idris A, Stiell I, Resuscitation Outcomes Consortium Investigators: Regional variation in out-of-hospital cardiac arrest incidence and outcome. JAMA 2008, 300:1423-1431.

63. Van de Werf F, Bax J, Betriu A, Blomstrom-Lundqvist C, Crea F, Falk V, Filippatos G, Fox K, Huber K, Kastrati A, Rosengren A, Steg PG, Tubaro M, Verheugt $F$, Weidinger F, Weis M, ESC Committee for Practice Guidelines $(\mathrm{CPG})$ : Management of acute myocardial infarction in patients presenting with persistent ST-segment elevation: the Task Force on the Management of ST-Segment Elevation Acute Myocardial Infarction of the European Society of Cardiology. Eur Heart J 2008, 29:2909-2945.

64. Cordell WH, Keene KK, Giles BK, Jones JB, Jones JH, Brizendine EJ: The high prevalence of pain in emergency medical care. Am J Emerg Med 2002, 20:165-169.

65. Berben SA, Meijs TH, van Grunsven PM, Schoonhoven L, van Achterberg T: Facilitators and barriers in pain management for trauma patients in the chain of emergency care. Injury 2012, 43:1397-1402.

66. Ebben $\mathrm{RH}$, Vloet $\mathrm{LC}$, de Groot JM, van Achterberg T: Factors influencing adherence to an emergency department national protocol. Eur J Emerg Med 2012, 19:53-56.

67. Lichtveld R, Adema R, Brendel R, Van der Werken C: Ambulancezorg van ernstig gewonde ongevalsslachtoffers: resulteert intensieve opleiding en toetsing in een beter resultaat voor de patiënt. Ned Tijdschr Traum 2006, 14:86-92.

68. Salerno SM, Wrenn KD, Slovis CM: Monitoring EMS protocol deviations: a useful quality assurance tool. Ann Emerg Med 1991, 20:1319-1324.

69. Baker R, Camosso-Stefinovic J, Gillies C, Shaw EJ, Cheater F, Flottorp S, Robertson N: Tailored interventions to overcome identified barriers to change: effects on professional practice and health care outcomes. Cochrane Database Syst Rev 2010, (3):CD005470. doi:CD005470

70. Daudelin DH, Sayah AJ, Kwong M, Restuccia MC, Porcaro WA, Ruthazer R, Goetz JD, Lane WM, Beshansky JR, Selker HP: Improving use of prehospital 12-lead ECG for early identification and treatment of acute coronary syndrome and ST-elevation myocardial infarction. Circ Cardiovasc Qual Outcomes 2010, 3:316-323.

71. Snider JB, Moreno R, Fuller DJ, Schmidt TA: The effect of simple interventions on paramedic aspirin administration rates. Prehosp Emerg Care 2004, 8:41-45.

72. Wollersheim H, Burgers J, Grol R: Clinical guidelines to improve patient care. Neth J Med 2005, 63:188-192.
73. Forsner T, Wistedt AA, Brommels M, Forsell Y: An approach to measure compliance to clinical guidelines in psychiatric care. BMC Psychiatry 2008, 8:64

74. MacFarlane C: The advances and evidence base for prehospital care. Emerg Med J 2003, 20:114-115.

75. Da Costa BR, Cevallos M, Altman DG, Rutjes AWS, Egger M: ses and misuses of the STROBE statement: bibliographic study. BMJ Open 2011 doi:10.1136/bmjopen-2010-000048. PubMed: http://www.ncbi.nlm.nih.gov/ pubmed/22021739.

doi:10.1186/1757-7241-21-9

Cite this article as: Ebben et al:: Adherence to guidelines and protocols in the prehospital and emergency care setting: a systematic review. Scandinavian Journal of Trauma, Resuscitation and Emergency Medicine $201321: 9$.

\section{Submit your next manuscript to BioMed Central and take full advantage of:}

- Convenient online submission

- Thorough peer review

- No space constraints or color figure charges

- Immediate publication on acceptance

- Inclusion in PubMed, CAS, Scopus and Google Scholar

- Research which is freely available for redistribution

Submit your manuscript at www.biomedcentral.com/submit
C Biomed Central 